\title{
Detection of plasmid-mediated AmpC $\beta$-lactamase in Escherichia coli
}

\author{
XIANGQUN LIU ${ }^{1}$ and YONGRUI LIU ${ }^{2}$ \\ ${ }^{1}$ Department of Respiration, Xuzhou City Hospital Affiliated to Xuzhou Medical College, Xuzhou, Jiangsu; \\ ${ }^{2}$ Department of Respiration, Jining No.1 People's Hospital, Jining, Shandong, P.R. China
}

Received February 2, 2016; Accepted April 20, 2016

DOI: $10.3892 /$ br.2016.661

\begin{abstract}
Escherichia coli (E. coli) is a common opportunistic pathogen for nosocomial infection. The aim of the study was to examine the phenotype, genotype and epidemiology of plasmid-mediated AmpC $\beta$-lactamases in E. coli. In total, 96 clinical isolates of repeated $E$. coli were collected from different hospitals between August and October 2012. Using a cefoxitin disk diffusion method to identify the phenotype of AmpC $\beta$-lactamases in E. coli, the plasmid was extracted, and multiplex polymerase chain reaction (PCR) was used to determine the amp gene. The PCR products were purified and sequenced. Of the 96 isolates strains, 43 strains were cefoxitin-resistant. Twelve $(12.5 \%)$ isolates were detected to produce AmpC $\beta$-lactamases with multiplex PCR, 11 strains carried DHA type ampC-resistant genes, and one strain carried ACC type ampC-resistant genes. In conclusion, the incidence of producing a plasmid-mediated AmpC enzyme of E. coli strains was relatively high. Therefore, antibiotics such as imipenem, a carbapenem, potentially serve as the treatment of choice for the infection.
\end{abstract}

\section{Introduction}

Escherichia coli (E. coli) is a common opportunistic pathogen for nosocomial infection (1). The National Antimicrobial Resistance Monitoring Network (www.carss.cn) has previously shown that the $E$. coli isolation rate ranked first as a gram-negative bacterium. This constitutes the reason for $\beta$ lactam antibiotic resistance producing the AmpC enzyme (2). The AmpC enzyme gene is transferred to other bacteria through conjugation and transformation, and is mediated by the plasmid. The AmpC enzyme-producing strains show broader resistance, making it challenging for clinicians to treat associated infections (3).

Correspondence to: Dr Xiangqun Liu, Department of Respiration, Xuzhou City Hospital Affiliated to Xuzhou Medical College, 19 Zhongshan North Road, Xuzhou, Jiangsu 221002, P.R. China E-mail: glxrui495638@163.com

Key words: Escherichia coli, plasmid, AmpC enzyme
In the present study, clinical isolates of $E$. coli-producing AmpC enzyme-mediated plasmid were examined in the local tertiary hospitals (Xuzhou City Hospital Affiliated to Xuzhou Medical College, Xuzhou No.1 People's Hospital and Xuzhou Central Hospital) between August and October 2012.

\section{Materials and methods}

Materials. In total, 96 strains of E. coli with no duplication of clinical specimens were collected from patients at the Xuzhou First People's Hospital, Affiliated Hospital of Xuzhou Medical College, Xuzhou Central Hospital (Jiangsu, China) between August and October 2012.

The standard AmpC-producing negative control strains were E. coli ATCC 25922. The positive control strains were Enterobacter cloacae 029M.

Instruments and reagents used to conduct the experiment were: Vitek-32 automatic microbiological analyzer (bioMerieux, Marcy l'Etoile, France), gel imaging system (Bio Canon, Shanghai, China), polymerase chain reaction (PCR) amplification instrument (Biometra $\mathrm{GmbH}$, Goettingen, Germany), electrophoresis apparatus (Beijing Liuyi Instrument Factory, Beijing, China), cefoxitin (FOX, $30 \mu \mathrm{g}$ ), and drug-sensitive slips. Mueller-Hinton agar was purchased from the British Opioid company (www.oxoid.com). Ex Taq enzyme, dNTPs, and DNA marker 100 were purchased from Tiangen Biotech Co., Ltd., Beijing, China. Agarose, and ethidium bromide were purchased from Sigma, St. Louis, MO, USA.

Method. Clinical specimens were cultured in blood agar medium at $35^{\circ} \mathrm{C}$ for $18-24 \mathrm{~h}$ and identified for bacteria and drug sensitivity analysis using the Microscan microbial identification system (Microscan, Siemens Healthcare Diagnostics, Deerfield, IL, USA). Primary screening of the AmpC enzyme-producing strains was conducted using the Kirby-Bauer disk diffusion method. Bacteria were detected using FOX $(30 \mu \mathrm{g})$ drug-sensitive slips according to the standards of USA National Committee for Clinical Laboratory Standards in 2009 (www. biomedresearch.com). A bacteriostatic circle diameter of $\leq 18 \mathrm{~mm}$ indicated strains producing AmpC enzyme.

The DNA of the positive clinical strains in the AmpC enzyme primary screening test was extracted using a bacterial DNA extraction kit, and this DNA served as a PCR template. The procedure was carried out according to the manufacturer's 
Table I. Polymerase chain reaction primer sequences and target gene.

\begin{tabular}{llc}
\hline Primers & \multicolumn{1}{c}{ Primer sequences $\left(5^{\prime} \rightarrow 3^{\prime}\right)$} & Expected product length (bp) \\
\hline Bla $_{\mathrm{MOx}}$ & P1: GCTGCTCAAGGAGCACAGGAT & 520 \\
& P2: CACATTGACATAGGTGTGC & \\
Bla $_{\mathrm{CIT}}$ & P1: TGGCCAGAACTGACAGGCAAA & 462 \\
& P2: TTTCTCCTGAACGTGGCTGGC & \\
$\mathrm{Bla}_{\mathrm{DHA}}$ & P1: AACTTTCACAGGTGTGCTGGGT & 405 \\
& P2: CCGTACGCTTACTGGCTTTGC & \\
$\mathrm{Bla}_{\mathrm{ACC}}$ & P1: AACAGCCTCAGCCGGTTA & 346 \\
$\mathrm{Bla}_{\mathrm{EBC}}$ & P2: TTCGCCGCAATCCCTAGC & \\
& P1: TCGGTAAAGCCGATGTTGCGG & 302 \\
$\mathrm{Bla}_{\mathrm{FOx}}$ & P2: CTTCCACTGCGGCTGCCAGTT & 190 \\
& P1: AACATGGGGTATCAGGGAGATG & \\
\hline
\end{tabular}

Table II. Twelve strains of AmpC enzyme producing Escherichia coli drug susceptibility results.

\begin{tabular}{|c|c|c|c|c|c|c|}
\hline \multirow[b]{2}{*}{ Antibiotics } & \multicolumn{2}{|c|}{ Sensitive } & \multicolumn{2}{|c|}{ Intermediate } & \multicolumn{2}{|c|}{ Resistance } \\
\hline & $\begin{array}{l}\text { No. of sensitive } \\
\text { strains }\end{array}$ & $\begin{array}{l}\text { The sensitive } \\
\text { rate }(\%)\end{array}$ & $\begin{array}{c}\text { No. of intermediate } \\
\text { strains }\end{array}$ & $\begin{array}{l}\text { The intermediate } \\
\text { rate }(\%)\end{array}$ & $\begin{array}{l}\text { No. of resistance } \\
\text { strains }\end{array}$ & $\begin{array}{c}\text { The resistance } \\
\text { rate }(\%)\end{array}$ \\
\hline FOX & 0 & 0.0 & 1 & 8.3 & 11 & 91.7 \\
\hline CRO & 3 & 25.0 & 1 & 8.3 & 8 & 66.7 \\
\hline CAZ & 1 & 8.3 & 1 & 8.3 & 10 & 83.3 \\
\hline FEP & 8 & 66.7 & 1 & 8.3 & 3 & 25.0 \\
\hline ATM & 1 & 8.3 & 1 & 8.3 & 10 & 83.3 \\
\hline $\mathrm{TZP}$ & 6 & 50.0 & 2 & 16.7 & 4 & 33.3 \\
\hline AMK & 8 & 66.7 & 1 & 8.3 & 3 & 25.0 \\
\hline CIP & 1 & 8.3 & 0 & 0.0 & 11 & 91.7 \\
\hline IVX & 1 & 8.3 & 0 & 0.0 & 11 & 91.7 \\
\hline IMP & 12 & 100.0 & 0 & 0.0 & 0 & 0.0 \\
\hline
\end{tabular}

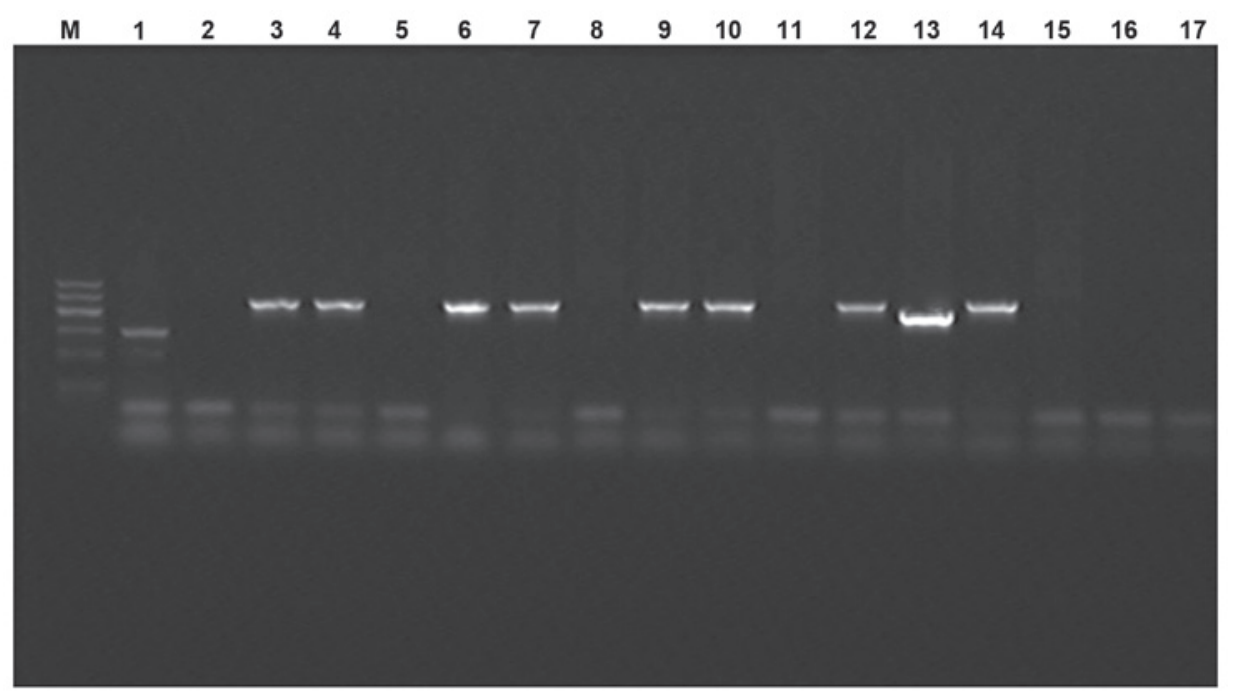

Figure 1. Electrophoresis of multiplex polymerase chain reaction (PCR) for detection of PCR products of AmpC enzyme producing gene mediated by plasmid. M, DNA marker (from top to bottom are 1,200, 900, 700, 500, 300 and $100 \mathrm{bp);} \mathrm{1,} \mathrm{positive} \mathrm{control;} \mathrm{2,} \mathrm{negative} \mathrm{control;} \mathrm{3-14,} \mathrm{Escherichia} \mathrm{coli}$ isolated from different clinic. 
protocol. The multiplex PCR primer sequences were carried out as previously described (1). Six pairs of primers were synthesized by Shanghai Saigon Biological Engineering Technology Co., Ltd. (Shanghai, China) (Table I).

The total volume of the reaction system was $50 \mu 1$. The PCR reaction conditions were: Pre-degeneration at $94^{\circ} \mathrm{C}$ for $3 \mathrm{~min}$, degeneration at $94^{\circ} \mathrm{C}$ for $30 \mathrm{sec}$, annealing at $56^{\circ} \mathrm{C}$ for $30 \mathrm{sec}$, extension at $72^{\circ} \mathrm{C}$ for $60 \mathrm{sec}$, for 28 cycles, and a final extension at $72^{\circ} \mathrm{C}$ for $7 \mathrm{~min}$. The PCR product underwent electrophoresis in $1.5 \%$ agarose gel containing $0.5 \mu \mathrm{g} / \mathrm{ml}$ ethidium bromide for gel imaging system observation and image recording.

The PCR products were analyzed by gene sequencing using bidirectional sequencing by Shanghai Saigon Biological Engineering Technology Co., Ltd. The results were assessed in BLAST to determine the genotype.

\section{Results}

The primary screening results identified that 43 of 96 strains tested for $E$. coli were resistant or intermediate to cefoxitin. The strains were AmpC enzyme-producing-positive strains in the primary screening. The positive rate was $44.8 \%$ (43/96).

The multiplex PCR results identified 12 cases that were positive, including 11 strains with an amplified fragment size of $\sim 405 \mathrm{bp}$. The strains were determined as type DHA according to the amplified fragment size. The amplified fragment size of the one strain was $\sim 346$ bp and was determined to be of type ACC. The total positive rate was $12.5 \%(12 / 96)$. The electrophoresis results are shown in Fig. 1. The purified PCR product sequencing results were crossed with GenBank database to determine the genotype of E. coli. Eleven strains of $E$. coli were type DHA with one strain of type ACC.

The results of $E$. coli producing AmpC enzyme mediated by plasmid were completely sensitive to imipenem with a high sensitivity to cefepime (66.7\%), amikacin (66.7\%) and a low sensitivity $(33.3 \%)$ to piperacillin and tazobactam.

The drug resistance rates to aztreonam, ciprofloxacin, and second- or third-generation cephalosporin were high (Table II).

\section{Discussion}

E. coli is an important pathogen in nosocomial infection (4). With the extensive application of broad-spectrum antibiotics, drug-resistant strains have been on the increase. AmpC enzyme production is the main mechanism for drug resistance. Although the AmpC structural gene exists in E. coli chromosome, it can only be produced from low levels of AmpC enzyme, due to a lack of the $A m p R$ gene. However, when E. coli obtains an exogenous $A m p C$ gene or the mutations lead to a high expression of AmpC structural gene, the result is resistance to many antibiotics. The AmpC enzyme-producing E. coli was recently identified (5). Thus, detection of the AmpC enzyme in $E$. coli in the tertiary hospital in the local region was useful in gaining a better understanding of the enzyme's prevalence and drug resistance.

The USA Committee for Clinical and Laboratory Standards Institute has not recommended use of a standard method for the detection of AmpC (6). Thus, the standard detection method for screening AmpC enzyme remains to be determined (7). The AmpC enzyme can hydrolyze cefoxitin whereas other $\beta$ lactam enzymes do not have this function. In the present study, 96 strains of $E$. coli were screened using a cefoxitin susceptibility disk test, and 43 strains were screened for producing AmpC enzyme. The positive E. coli in the primary screening were detected the genotypes using multiplex PCR technique (8). Twelve strains of E. coli amplification were positive for the test, including 11 strains of tested bacteria amplified bands of $\sim 405$ bp in size that were identified as DHA type AmpC enzymes according to the size of the fragments. One strain of tested bacterial amplified bands of 346 bp in size was determined to be the ACC type AmpC enzyme according to the fragment size. The total positive rate of the E. coli-producing AmpC enzyme mediated by plasmid in the tertiary hospital in the Xuzhou region was $12.5 \%$. Dong et al (9) have reported that the enzyme may be associated with the widely used third-generation antibiotics in this region. The most common $\mathrm{AmpC}$ resistance gene is type DHA-1 and type ACT. The present findings have shown that the E. coli-producing AmpC enzyme mediated by plasmid is mainly the DHA type in the tertiary Hospital of Xuzhou region, which is consistent with previous studies (10). Thus, type DHA may be the main base of domestic plasmid-mediated AmpC enzyme combined with the results of the present study.

The findings have shown that the drug susceptibility of AmpC enzyme-producing E.coli exhibited multiple drug resistance to second- or third-generation cephalosporin including cefoxitin, ceftazidime, and ceftriaxone. It also showed high resistant to quinolone antibiotics such as ciprofloxacin and levofloxacin. The Amikacin drug resistance rate was $25 \%$ with a relatively low resistance rate of $25 \%$. The fourth-generation cephalosporin was recommended for the treatment infection of strains producing $\mathrm{AmpC}$ as it rapidly passes through the outer membrane barrier. The E. coli producing the AmpC enzyme in the present study was sensitive to cefepime. Therefore, the fourth-generation cephalosporin can be used for the treatment of related infection. Additionally, E. coli resistance to carbapenem antibiotic imipenem in the present study is due to the high stability of carbapenem antibiotics to $\beta$-lactamase. Carbapenem antibiotic imipenem is thus recommended for the clinical treatment of AmpC enzyme-producing $E$. coli infection mediated by plasmid. The application of the fourth-generation cephalosporins and aminoglycosides for treatment may also be considered.

The present study has demonstrated that the detection rate of the AmpC enzyme-producing E. coli was high in the tertiary hospital of the Xuzhou area. Carbapenem antibiotics are thus a suitable choice for treatment of related infection.

\section{References}

1. Weinstein RA: Nosocomial infection update. Emerg Infect Dis 4: 416-420, 1998.

2. Jacoby GA: AmpC beta-lactamases. Clin Microbiol Rev 22: 161-182, 2009.

3. Markovska R, Schneider I, Marteva-Proevsk Y, Mitov I, Bauernfeind A and Markova B: First detection of the AmpC beta-lactamase ACC-1 in a Klebsiella pneumoniae isolate in Bulgaria. J Chemother 24: 307-308, 2012.

4. Ma XZ, Lv Y and Xue F: Ministry of health, the nationalantimicrobial resistance monitoring report: Bacterial drug resistance monitoring bloodstream infection for 2010 . Chin J Nosocomiol 21: 5147-5151, 2011 (In Chinese). 
5. Ahmed SF, Ali MM, Mohamed ZK, Moussa TA and Klena JD Fecal carriage of extended-spectrum beta-lactamases and AmpC-producing Escherichia coli in a Libyan community. Ann Clin Microbiol Antimicrob 13: 22. 2014.

6. Clinical and Laboratory Standards Institute: Performance standards for antimicrobial susceptibility testing. Sixteenth informational supplement. M100-S116, 2006.

7. Joseph NM and Mathias S: Challenges in detection of AmpC $\beta$-lactamases among Enterobacteriaceae. Indian J Med Res 137: 216-217, 2013.
8. Yilmaz NO, Agus N, Bozcal E, Oner O and Uzel A: Detection of plasmid-mediated AmpC $\beta$-lactamase in Escherichia coli and Klebsiella pneumoniae. Indian J Med Microbiol 31: 53-59, 2013.

9. Dong F, Xu X and Song W: Study on AmpC beta lactamases in clinical isolates of Escherichia coli and Klebsiella plasmid mediated. Chin Med J (Engl) 90: 2723-2725, 2010.

10. Ying Ye, Qian Wang, Yan Chen and Jiabin Li: Identification of plasmid-mediated AmpC $\beta$-lactamase in Escherichia coli. Chin J Lab Med 30: 662-665, 2007. 